\title{
A high-resolution method for estimating the azimuth of infrasound signals
}

\author{
Liang Meng, Xihai Li,Dingxin Chen and Tingting Yang \\ Xi'an Research Institute of High Technology, Xi'an 710025, People's Republic of China \\ ml7290@sohu.com
}

Keywords:infrasound signals, FK, MUSIC, azimuth

\begin{abstract}
The work described herein discusses the application of a high-resolution frequency wavenumber method-the Multiple Signal Classification (MUSIC) method which is different from the traditional frequency wavenumber (F-K) method for detectingand estimating the direction of travel (DOT) of infrasound. Synthetic infrasound signals were superposed on top of white noise to assess the performance of the two algorithms with respect to signal-to-noise ratio. The synthetic array is composed of four elements that are aligned and equally spaced on a line. Preliminary results demonstrate the MUSIC method can lead to more accurate DOT detection than the F-K method.
\end{abstract}

\section{Introduction}

The work described in this paper is a high-resolution method-Multiple Signal Classification (MUSIC) method for detecting infrasonic signals and determining their direction of travel (DOT).The F-K technique was used before to detect and determine DOT of infrasound.A description of the F-K technique can be found, e.g., in Capon[1](1969) or as a larger review in Capon[2](1973). This method has been further developed to include wide-band, maximum-likelihood estimation techniques, and three-component data[3-5].Costley[6] used the F-K technique for signals from rectangular infrasound arrays for detection and estimation of the DOT of infrasound.

The MUSIC algorithm is a high-resolution frequency wavenumber method. In mobile communication, MUSIC has been the most widely used for directional of arrival (DOA) estimation. [The different between DOT and DOA is $180^{\circ}$ ]. In a detailed performance evaluation based on hundreds of simulations, MIT's Lincoln Laboratory concluded that among high-resolution algorithms then available, MUSIC was the most promising[7].

In this paper, the high-resolution MUSIC method is applied to determine the DOA of infrasound in the CTBT interesting scale and presents the performance analysis and comparison for FK and MUSIC DOAestimation algorithms. In order to assess the performance of the two algorithms, synthetic infrasound signals were superposed onto white noise.

\section{The Music algorithm}

Assume an M-element array of sensors, P narrowband far-field signal sources, and define $A(\theta)$ to be the complex array response for a source with DOA $\theta$.

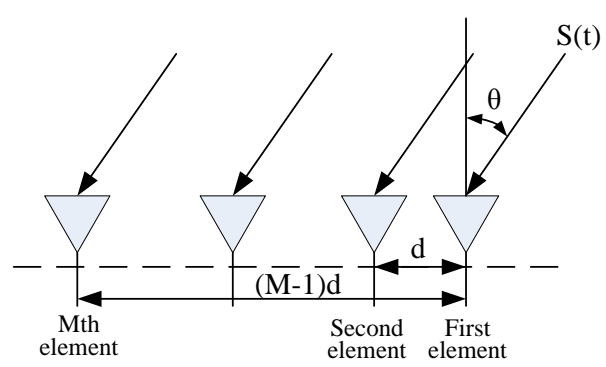

Figure 1. Uniform linear array 
The outputs of the $\mathrm{M}$ array elements at time $t$ are stacked in a vector $X(t)$. Under the assumptions that the signals waveforms are narrowband and that the array elements are linear devices, the array output $X(t)$ can be written as

$$
X(t)=A S(t)+n(t)
$$

Where $S(t)$ is the amplitude and phase of the signals at time $t, n(t)$ is additive noise, and where

$$
A=\left[a\left(\theta_{1}\right), a\left(\theta_{2}\right), a\left(\theta_{3}\right) \mathbf{L} a\left(\theta_{P}\right)\right]
$$

The number of signal eigenvalues and eigenvectors is $\mathrm{P}$ and number of noise eigenvalues and eigenvectors is M-P. The array correlation matrix with uncorrelated noise and equal variances is then given by

$$
R_{X X}=A^{*} R_{S S} * A^{H}+\sigma_{n}^{2} * I
$$

where $\quad R_{S S}=\left[s_{1}(k), s_{2}(k), s_{3}(k) \mathbf{L} s_{P}(k)\right]^{T} . \quad R_{X X}$ has $\mathbf{P}$ eigenvectors associated with signals and M-P eigenvectors associated with the noise. We can then construct the $M \times(M-D)$ subspace spanned by the noise eigenvectors such that

$$
V_{N}=\left[v_{1}, v_{2}, v_{3} \mathrm{~L} v_{M-D}\right]
$$

The noise subspace eigenvectors are orthogonal to array steering vectors at the angles of arrivals $\theta_{1}, \theta_{2}, \theta_{3}\left\llcorner\theta_{P}\right.$ and the MUSIC pseudospectrum is given as

$$
P_{M U S I C(\theta)}=1 / a b s\left(a(\theta)^{H} * V_{N} * V_{N}^{H} * a(\theta)\right)
$$

\section{The F-K process}

According to Smart and Flinn, the F-K spectrum can be calculated in a single summation. With the relation $K=w P$ between the slowness vector $P$ and the wavenumber $K$ for frequency $w$, the F-K spectrum is converted to frequency-slowness power spectrum:

$$
P(w, p)=\left|\sum_{n=1}^{N} A_{n}(w) \cdot e^{-i w p r_{n}}\right|^{2}
$$

With: $A_{n}(w)$ being the Fourier transforms of recordings at M number of instruments located by $r_{n}$ position vectors. $P(w, p)$ can be interpreted as a set of beams sampling the atmosphere above the array. In this paper, we assume the speed of the infrasound wave is known, but the DOA is not known. The F-K and MUSIC algorithms for DOAestimation are compared.

\section{Process with synthetic infrasonic signals}

The simulated Gaussian-modulated sinusoidal pulses added to white noise. The SNR is determined as the ratio of the magnitudes of the pressure spectra of pure signal and pure noise in the $2 \mathrm{~Hz}$. We assume the array is composed of four elements that are aligned and equally spaced on a line. The spacing between neighboring elements is $85 \mathrm{~m}(\lambda / 2)$. The appropriate phase is added to the simulated signal recorded at each sensor to represent an acoustic signal with a DOA of $30^{\circ}$ and sound speed of $u=340 \mathrm{~m} / \mathrm{s}$. The simulated signals are plotted in Figure 2. The FK and MUSIC spectrum for different values of SNR are plotted in Figs.3 (a)-(c). The spectrums of the two methods are overlapped for comparison.

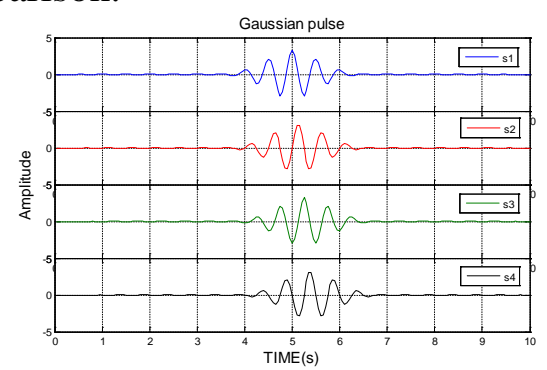

Figure 2.The traces represent the simulated Gaussian-modulated sinusoidal pulse, with delays to simulate propagation over array. 

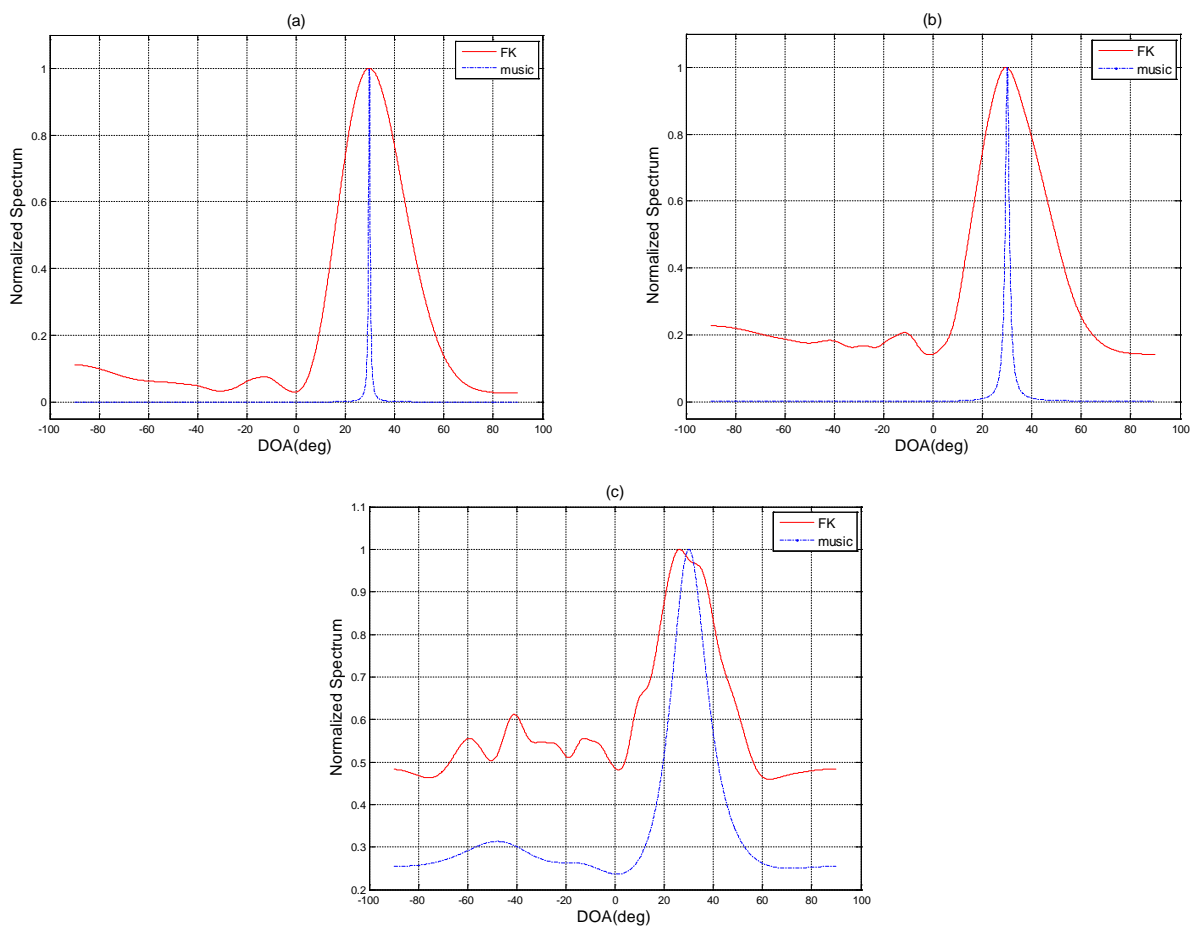

Figure 3(a)-(c) represent the spectrum when $\mathrm{SNR}=10 \mathrm{~dB}, 0 \mathrm{~dB}$ and $-10 \mathrm{~dB}$, respectively.

Figs.3 shows that MUSIC spectrum takes the form ofsharper peaks in which angular resolution is improved, especially for $\mathrm{SNR}=10 \mathrm{~dB}$, and $0 \mathrm{~dB}$. So MUSIC spectrum can lead to more accurate detection. To prove this result, Root mean square error (RMSE) is used as the metric to compare the performance of the two algorithms.Figure 4 shows the RMSE in DOA estimation as a function of SNR for the two algorithms. The RMSE is average over 20 repeated DOA estimation trials.

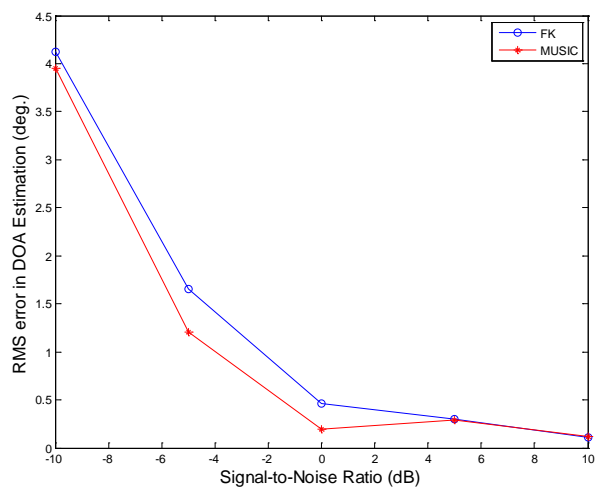

Figure 4.The RMSE in DOA estimation as a function of SNR for the two algorithms

As seen from Figure 4, the two algorithms perform about equally well when the SNR is high. Under low SNR conditions it is seen that the method of MUSIC performs better than the FK method, especially for $\mathrm{SNR}=0 \mathrm{~dB}$ and $-5 \mathrm{~dB}$.

\section{Discussion and conclusions}

A high-resolution method for processing distributed arrays of infrasound sensors was presented. The performance of the method was compared to the F-K technique. For linear array, the MUSIC spectrum took the form ofsharper peaks than the F-K's, and the RMSE of the MUSIC spectrum was smaller than the F-K's, especially for $S N R=0 \mathrm{~dB}$ and $-5 \mathrm{~dB}$. So the performance of the MUSIC method is better than the F-K, and the MUSIC spectrum can lead to more accurate DOA detection. 


\section{References}

[1]. J. Capon, “High-resolution frequency-wavenumber spectrum analysis,” Proc. IEEE,57, 1408-1418(1969).

[2]. J. Capon, “ Signal Processing and frequency-wavenumber spectrum analysis for a largeaperture seismic array,” Methods in computational physics, 1-59(1973).

[3]. T. Kværna, and D. J. Doornbos, “An integrated approach to slowness analysis with arrays and three-component stations,” NORSAR Sci. Rep., 2-85/86, 60-69(1986).

[4]. T. Kværna, and F. Ringdal, "Stability of various f-k estimation techniques," NORSAR Sci. Rep., 1-86/87, 29-40(1986).

[5]. E. Ødegaard, D. J. Doornbos, and T. Kværna, “Surface topographic effects at arrays and three-component stations,” Bull. Seism. Soc. Am., 80, 2214-2226(1990).

[6]. R. D. Costley, W. G. Frazier, K. Dillion, J. R. Picucci, J. E. Williams, "Frequency-wavenumber processing for infrasound distributed arrays," J. Acoust. Soc. Am., 134(4), 307-313(2013).

[7]. A. J. Barabell, J. Capon, D. F. Delong, J. R. Johnson, and K. Senne, "Performance Comparison of Superresolution Array Processing Algorithms," Technical Report TST-72, Lincoln Laboratory, M.I.T.(1984). 